\title{
$\begin{array}{ll}\text { Research Square } & \text { Preprints are preliminary reports that have not undergone peer review. } \\ \text { They should not be considered conclusive, used to inform clinical practice, }\end{array}$ \\ or referenced by the media as validated information. \\ Three-Dimensional Transient Finite Element Cooling Simulation For Injection Molding Tools
}

\section{Lu Chen}

Huazhong University of Science and Technology

\section{Xiaowei Zhou}

Huazhong University of Science and Technology

Zhigao Huang ( $\nabla$ huangzhigao@hust.edu.cn )

Huazhong University of Science and Technology

Huamin Zhou

Huazhong University of Science and Technology

\section{Research Article}

Keywords: Injection molding, Mold cooling, FEM, Transient cooling simulation

Posted Date: October 18th, 2021

DOI: https://doi.org/10.21203/rs.3.rs-969469/v1

License: (c) (1) This work is licensed under a Creative Commons Attribution 4.0 International License. Read Full License 


\title{
Three-Dimensional Transient Finite Element Cooling Simulation for Injection Molding Tools
}

\author{
Lu Chen, Xiaowei Zhou, Zhigao Huang*, Huamin Zhou \\ State Key Laboratory of Materials Processing and Die \& Mould Technology, School of Materials \\ Science and Engineering, Huazhong University of Science and Technology, Wuhan 430074, China. \\ ${ }^{*}$ Corresponding author. Tel.: +86 2787543492. Fax: +86 2787554405. E-mail address: \\ huangzhigao@ hust.edu.cn
}

\begin{abstract}
Plastic injection molding is one of the most popular manufacturing processes for mass production, and optimizing the mold cooling system is critical for reducing the cycle time and improving the final part quality. Conventional cooling simulation uses the boundary element method to perform the cycle-averaged analysis, which is a simplification due to computational resources limitation. This paper develops a three-dimensional transient cooling simulation method based on the finite element method, which can simulate the complex mold system accurately and efficiently. It is shown that this method finishes the transient cooling analysis in 478 seconds on the real-world injection molding mold with more than 6.9 million tetrahedral elements. Its accuracy is compared against the experimental results with the maximum temperature error less than $4 \%$, and the average temperature error less than $1 \%$.
\end{abstract}

Keywords: Injection molding, Mold cooling, FEM, Transient cooling simulation

\section{Introduction}

Plastic injection molding is one of the most popular manufacturing processes for mass production. The typical injection molding stages are: filling and packing stage, cooling stage and mold open stage. The mold temperature within the injection molding cycle is transient in nature, which increases at the filling stage and decreases at the subsequent stages. Among all these molding stages, cooling stage is of particular importance as it takes more than $70 \%$ of the entire cycle time, hence the cooling system of the mold tool plays a crucial role as it has huge impact to both of the cycle time and part quality. Indeed, a proper cooling system design can significantly reduce the cost by shortening the cycle time, and avoid the final part defects such as warpage and sink marks [1].

In order to obtain cost effective and high qualitied parts consistently, many molders turn to simulation technology for part and mold design. The injection molding cooling simulation aims to provide the mold designers with accurate mold cooling results to guide their design decisions, far before the molds are actually manufactured. The earliest while most wildly used mold cooling simulation method is the boundary element method (BEM) based cycle-averaged cooling model [2]. Due to the thin wall nature of the injection parts, a finite difference discretization method over the 
thickness direction for the 2.5D parts was developed [3]. Then the BEM based cycle-averaged cooling model extended the part to three-dimensional mesh [4-7], and more BEM based researches were reported [8-13]. The cycle-averaged model is a steady state solution that approximates the transient nature within the injection molding cycle. To simulate the transient cooling effect, SY Hu et al. [14] used dual reciprocity boundary element method (DRBEM) to study the mold transient heat transfer characteristics. Tang et al. [15] used finite element method (FEM) to solve the transient mold temperature starting from a uniform initial mold temperature.

The BEM based cycle-averaged cooling model is slow to solve on large models. The BEM method reduces the mold from solid domain to the surface domain, hence the discretization and computation are only carried out at the surfaces, without the need to generate solid mesh. However, the cost of reducing dimension is not trivial, the BEM generates a full dense matrix. The dense matrix from BEM is more difficult to solve and requires more memory than the sparse matrix formed by FEM [16]. This even deteriorates with the state-of-the-art simulation software, as the mesh tends to be several orders finer than before due to the advancement of the computer hardware, which allows mold designers to chase higher simulation accuracy. Such fine mesh generates huge matrix for BEM to solve. It is always observable that the cooling analysis takes much longer than the flow analysis on those complex parts. To speed up the BEM cooling simulation, Zhou et al. [1] proposed an acceleration algorithm.

The BEM doesn't support simulating the real-world three-dimensional mold. It uses a box to represent the actual mold, which is a huge simplification and loses many details. Although this is acceptable from the engineering perspective in many cases, such simplifications cause difficulties on simulating advanced features such as the heat resistance between multiple mold plates, hot runner system, heater cartridge system, or special mold insert such as the 3D printed conformal cooling channel insert.

The cycle-averaged mold temperature assumption is not always true. It assumes the mold temperature doesn't change significantly during the injection molding cycle, but there are exceptions. For some deep hollowed parts such as the car lights, the conventional cooling channels are difficult to cool down the mold core side, so the mold core temperature can have large variation within the cycle. Other cases such as the two shot overmolding process [17] and rapid heating and cooling process [18], the cycle-averaged assumption no longer holds. Even for the conventional injection molds, the transient mold temperature is still more accurate than the cycle-averaged solution. The reason why using cycle-averaged model is because the transient analysis takes much longer time to run. The transient mold temperature should be used if the transient cooling analysis runs with acceptable performance.

The objective of this paper is to develop a three-dimensional transient FEM cooling algorithm for the injection molding process. This work has three major highlights: First, the performance issue of conventional BEM is improved by using FEM. FEM generates sparse positive definite matrix which can be solved more efficiently than the dense matrix from BEM. Second, this work allows 
using three-dimensional mold mesh to describe the mold system without any compromises, the mold designers can start the cooling simulation directly from the complete CAD models of mold and plastic part, hence a true three-dimensional simulation over both part and mold can be performed, and it allows for arbitrary number of separate mold components with different mesh sizes to be solved simultaneously. Third, a more accurate transient cooling simulation can be performed efficiently. This work employs the cycle-averaged mold temperature solution as the initial condition for the transient cooling analysis, and it converges much faster than the uniformed initial mold temperature that is used in [15].

This paper is organized as below. First the modeling assumptions are given in chapter 2. In chapter 3 , the governing equations, boundary conditions, and the numerical implementation are discussed. In chapter 4 an initial condition for speeding up the transient cooling analysis is given, and chapter 5 discusses a special boundary condition treatment for the mold-mold boundary and mold-polymer boundary. In chapter 6 the experiment and simulation setup are given for validation purpose, then the simulation results are compared against the experimental results in chapter 7 .

\section{Modeling and basic assumption}

As mentioned in chapter 1 that the typical injection molding stages are filling and packing stage, cooling stage and mold open stage. The melt polymer is injected into the mold cavity during filling stage which heats up the mold, and the cooling system keeps pumping coolant into the mold to bring the mold temperature down. At the very beginning of the production, the mold is completely cold at the surrounding temperature. After multiple injection cycles, there exists a heat balance such that the mold temperatures curves are similar between cycles, and the production runs under such stable injection molding cycles. In figure 1, the transient mold temperature gradually builds up from the beginning of the injection production, after a number of injection molding cycles, the temperature enters a stable cyclic state, in which the mold temperature varies within the cycle but all the injection molding cycles are the same [2]. The cycle averaged mold temperature, the red line in the curve, is the averaged value of such stable cycles. Conventional cooling simulation calculates this cycle-averaged mold temperature, but the aim of this work is to quickly solve the transient mold temperatures under such stable cycles. 


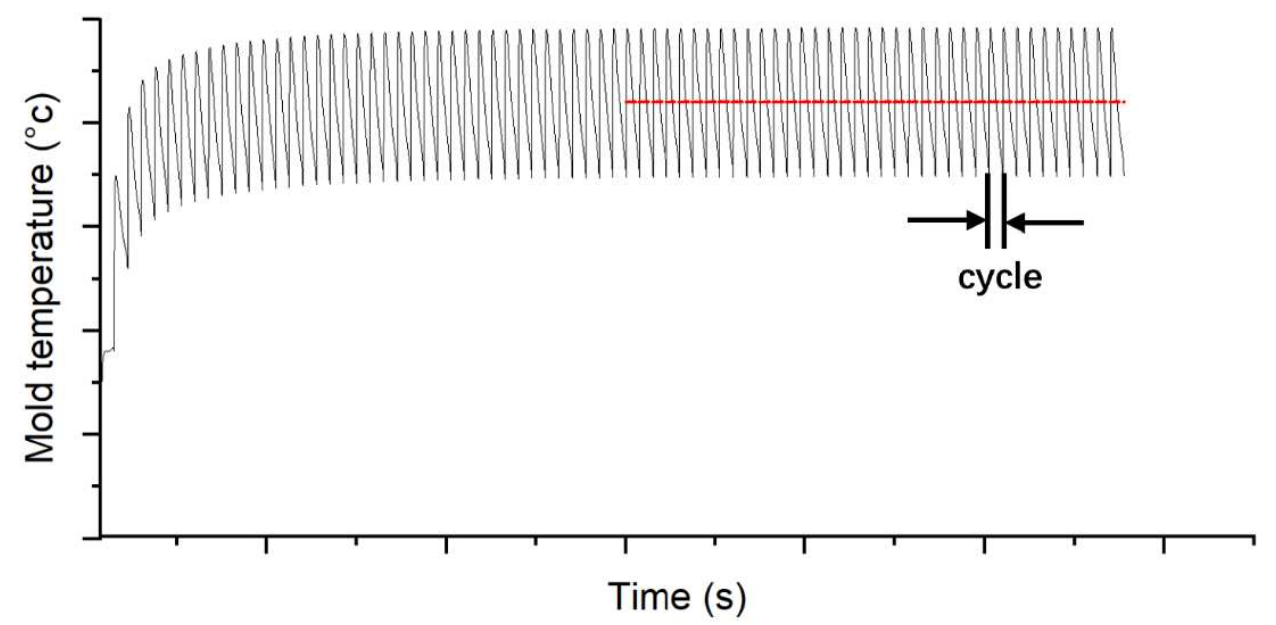

Figure 1. Transient mold temperature builds up

From the energy conservation point of view, the injection mold absorbs heat from part through the mold-polymer boundary, and leaks heat into the coolant and surrounding through the moldcoolant boundary and mold-air boundary as shown in figure 2 . These effects can be regarded as heat exchange with the external system of the mold. Inside the mold system, there are also multiple mold components which are closely contacted to each other, such as the mold-mold boundary in figure 2 . These mold platens contact boundaries usually are neglected in BEM by assuming the mold is a simple box, but the real mold is a complex assembly with many separate components. These mold components may not always in perfect contact, there can be gaps or grease in between. Hence the thermal conduction through the contact interface can be less efficient than the perfect contact situation.

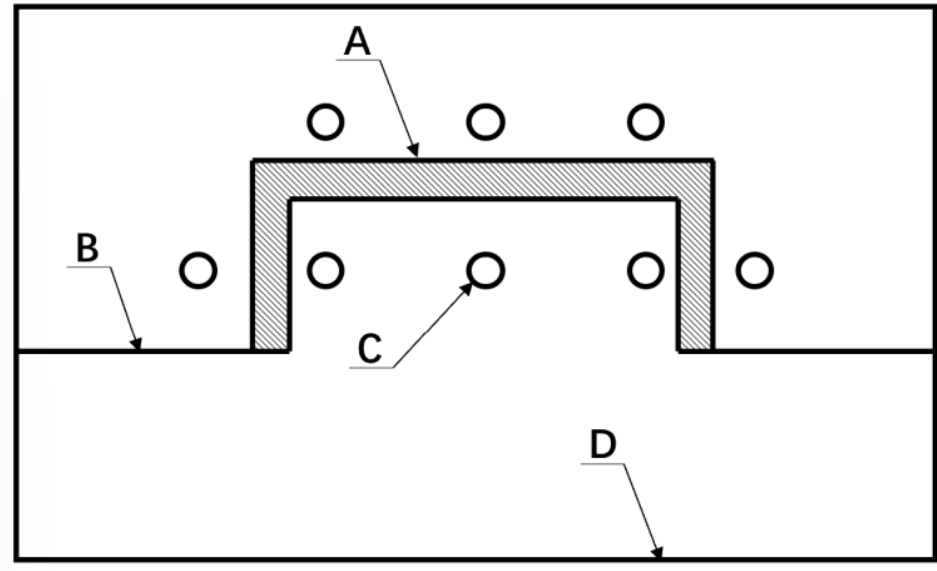
A - mold-polymer boundary
B - mold-mold boundary
C - mold-coolant boundary
D - mold-air boundary

Figure 2. Different types of mold boundaries

The filling time is usually very short comparing with the packing time, cooling time and mold 
open time, and the filling stage can be simplified by assuming the melt polymer is full of the cavity at the beginning of the injection molding cycle. Hence the convection effect of the melt polymer can be ignored and only the heat conduction effect needs to be addressed, which greatly simplified the governing equation.

From the transient cooling simulation perspective, the governing equations of the whole computational domain, initial conditions and boundary conditions are needed to solve the problem. Figure 3 shows this transient cooling simulation workflow (B), and also compares against the conventional cooling simulation workflow (A). It can see that there are two major differences in this work. First, the part and mold coupling loop is not needed, which can significantly improve the simulation speed, accuracy and avoid the numerical convergence issues during the part and mold iterations. Apart from that, it also allows solving arbitrary number of separate mold blocks, hence this work can solve the complex CAD mold models directly. Second, there is one extra step which solves the initial condition for this transient cooling analysis, the aim of this step is to find a better initial value for the transient analysis to speed up the convergence.

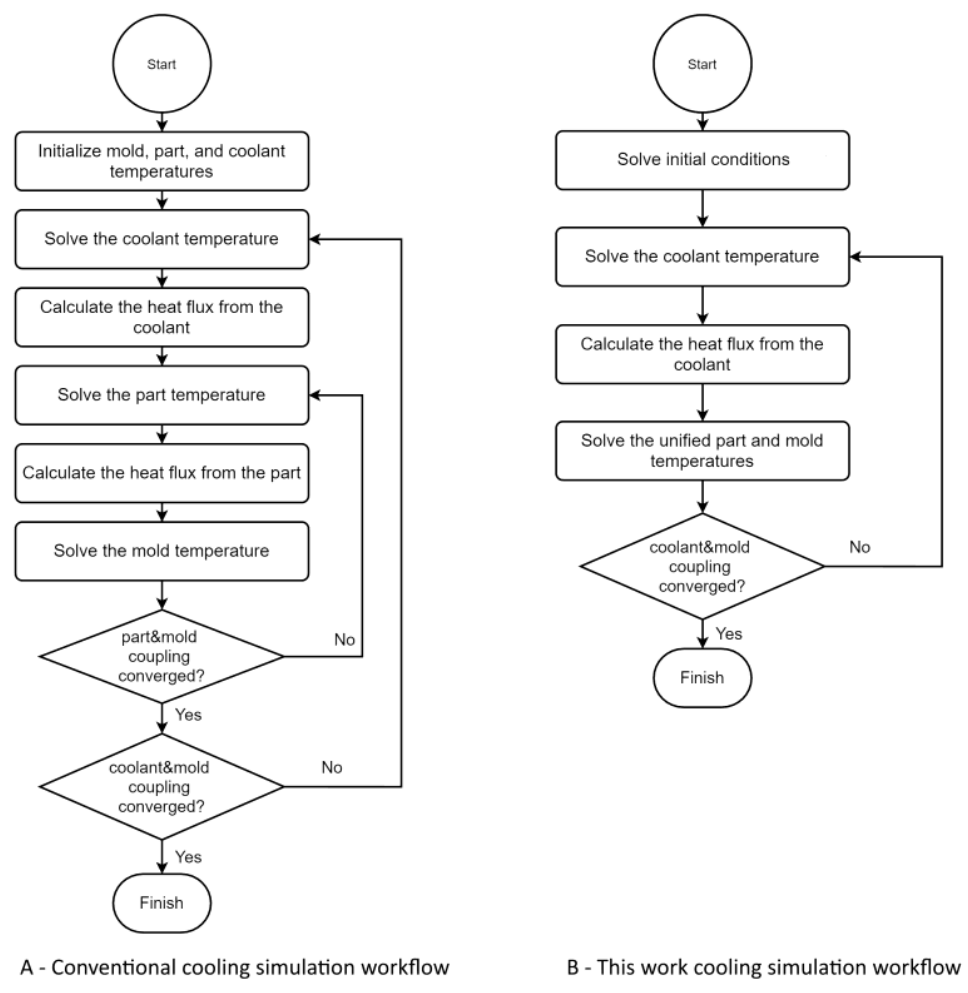

Figure 3. Workflows of the conventional cooling simulation and this work

\section{Transient FEM cooling formulation}

\subsection{Governing equation}

As the filling stage of the melt polymer has been neglected, only the transient effect and conduction effect need to be considered, hence the part and mold share the same governing equation, which is given in equation (1) [15]. 


$$
\rho C_{p} \frac{\partial T}{\partial t}=\nabla \cdot(k \nabla T)
$$

In Equations (1) above, $\rho$ is the density, $C_{p}$ is the specific heat, $k$ is the thermal conductivity, $t$ is the time, and $T$ is the temperature. The material properties $\rho, C_{p}$, and $k$ are different for polymers and mold.

By applying FEM to discretize equation (1), we can obtain the discretized form for one element at time step $\mathrm{k}$ which is shown in equation (2). The $\left\{T^{k}\right\}$ is element nodal temperature unknowns at time step $\mathrm{k}$, The $\left\{T^{k-1}\right\}$ is element nodal temperature unknowns of time step k-1 which are solved at last time step, $\boldsymbol{N}$ is the element shape functions vector.

$$
\int\left(\frac{\rho C_{p}}{\Delta t} \boldsymbol{N}^{T} \boldsymbol{N}+k \nabla \boldsymbol{N}^{T} \nabla \boldsymbol{N}\right) d V \cdot\left\{T^{k}\right\}=\int \frac{\rho C_{p}}{\Delta t} \boldsymbol{N}^{T} d V \cdot\left\{T^{k-1}\right\}
$$

\subsection{Mold-polymer boundary condition}

Conventional mold-polymer boundary condition applied to the mold side is done via an iterative approach, as shown on the left side of figure 3 . At the beginning of the analysis, the mold is assumed at a guessed temperature, such guessed mold temperature is used as boundary condition to solve the part temperature, then the heat flux is calculated from part side and used as boundary condition to solve the mold temperature, by running this loop until the mold temperature variation and part heat flux variation are small enough, then the part and mold temperatures are thought to be converged. This is a slow process, and the mold temperature variation or part heat flux variation may never converge on complex models. The reason is that the mold mesh and part mesh are separated meshes, they are not connected at the interfaces hence the heat can't pass through from one mesh to the other, then an iterative method must be performed to reach the heat flux balance at the mold-polymer boundary.

This paper develops a method to avoid the iterative way at the mold-polymer boundary. According to the work of Liu et al. [19], at the mold-polymer interface, the mold temperature isn't exactly equal to part temperature, there is a heat transfer coefficient $h_{p}$ between the part and mold. The heat flux at the mold-polymer interface $q_{p}$ can be defined in equation (3), in which $T_{m}$ is the mold temperature and $T_{p}$ is the part temperature.

$$
q_{p}=h_{p}\left(T_{p}-T_{m}\right)
$$

In equation (3), both the $T_{m}$ and $T_{p}$ are unknown hence it can't be treated as normal thermal boundary condition. Rewrite equation (2) into the matrix form, and separate mold nodal temperatures and part nodal temperatures, we can have the equation (4). $K_{p}$ is the thermal system matrix over the entire part domain, $K_{m}$ is the thermal system matrix over the entire mold domain, $T_{p}$ is the part temperature unknowns, $T_{m}$ is the mold temperature unknowns, $r h s_{p}$ and $r h s_{m}$ 
are right hand side known values for part and mold.

$$
\left[\begin{array}{cc}
K_{p} & 0 \\
0 & K_{m}
\end{array}\right]\left\{\begin{array}{c}
T_{p} \\
T_{m}
\end{array}\right\}=\left\{\begin{array}{l}
r h s_{p} \\
r h s_{m}
\end{array}\right\}
$$

By substituting equation (3) into (4), we can have the mold-polymer boundary constrained system equation (5). $K_{p m}$ stands for the mold-polymer boundary constraints for part nodes, and $K_{m p}$ stands for the mold-polymer boundary constraints for mold nodes. $K_{p m}$ and $K_{m p}$ are not necessarily equal. It depends on the mesh at the boundary and will be discussed in chapter 5 . The original $K_{p}$ and $K_{m}$ now becomes $K_{p}{ }^{\prime}$ and $K_{m}{ }^{\prime}$ as the equation (3) also adds up to coefficients to them.

$$
\left[\begin{array}{cc}
K_{p}{ }^{\prime} & -K_{p m} \\
-K_{m p} & K_{m}{ }^{\prime}
\end{array}\right]\left\{\begin{array}{c}
T_{p} \\
T_{m}
\end{array}\right\}=\left\{\begin{array}{l}
r h s_{p} \\
r h s_{m}
\end{array}\right\}
$$

With the equation (5), the mold and part temperatures can be solved simultaneously in one single system matrix without any iterative method needed.

\subsection{Mold-mold boundary condition}

The real injection mold is never simple, it consists of hundreds of components. Even after excluding unimportant components, there are still multiple important mold platens for simulation. Similar with section 3.2, since these mold platens are separate CAD bodies and separate meshes as well, the best way to handle the mold-mold boundary condition is to apply equation (6) into equation (5). $h_{m_{i j}}$ is the heat transfer coefficient between the mold platen i and mold platen $\mathrm{j}, T_{m_{i}}$ and $T_{m_{j}}$ are the mold temperatures of the mold platen i and mold platen $\mathrm{j} . h_{m_{i j}}$ is not trivial as the mold platens may not always in perfect contact. There might be grease, gaps, or some intentionally added insulating layer between mold platens.

$$
q_{m_{i j}}=h_{m_{i j}}\left(T_{m_{i}}-T_{m_{j}}\right)
$$

Similar with the treatment in section 3.1. By substituting equation (6) into (5), we can obtain system equation (7) with mold-mold boundary condition handled.

$$
\left[\begin{array}{ccc}
K_{p}{ }^{\prime} & -K_{p m_{i}} & -K_{p m_{j}} \\
-K_{m_{i} p} & K_{m_{i}}{ }^{\prime} & -K_{m_{i} m_{j}} \\
-K_{m_{j} p} & -K_{m_{j} m_{i}} & K_{m_{j}}{ }^{\prime}
\end{array}\right]\left\{\begin{array}{c}
T_{p} \\
T_{m_{i}} \\
T_{m_{j}}
\end{array}\right\}=\left\{\begin{array}{c}
r h s_{p} \\
r h s_{m_{i}} \\
r h s_{m_{j}}
\end{array}\right\}
$$

\subsection{Mold-air boundary condition}

At the mold-air boundary, the boundary condition is defined in equation (8), in which a heat transfer coefficient $h_{a}$ between the mold and air. The heat dissipates into the air isn't major in cooling simulation and $h_{a}=10 \mathrm{~W} \cdot \mathrm{K}^{-1} \cdot \mathrm{m}^{-2}$ is a good engineering approximation. $T_{a}$ is assumed to be a known constant. 


$$
q_{a}=h_{a}\left(T_{m}-T_{a}\right)
$$

\subsection{Mold-coolant boundary condition}

At the mold-coolant boundary, the boundary condition is defined in equation (9), in which a heat transfer coefficient $h_{c}$ between the mold and coolant [1] is defined in equation (10) to approximate the cooling effect of the cooling system. The $q_{c}$ is the heat flux at the mold-coolant boundary, $T_{m}$ is the mold temperature, $T_{c}$ is the coolant temperature, $R e$ is the Reynolds number, $\operatorname{Pr}$ is the Prandtl number, $k_{c}$ is the thermal conductivity of the coolant, and $\mathrm{D}$ is the diameter of the cooling channel.

$$
\begin{gathered}
q_{c}=h_{c}\left(T_{m}-T_{c}\right) \\
h_{c}=0.023 \frac{k_{c}}{D} \operatorname{Re}^{0.8} \operatorname{Pr}^{0.4}
\end{gathered}
$$

\section{Initial condition for transient cooling}

The transient cooling simulation governed by equation (1) can start from an initial temperature and update temperature field step by step for many injection cycles, until the stable cycle reaches as shown in figure 1 . However, starting from an arbitrary initial value can make the mold temperature to reach the stable cycle slowly. A good initial value for mold temperature can significantly improve the convergence of the simulation. This chapter develops the three-dimensional FEM based cycleaveraged steady state mold temperature as the initial value for the transient cooling analysis.

The governing equation for the cycle-averaged model for the mold side is given in equation (11). $k_{m}$ is the thermal conductivity of the mold.

$$
\nabla \cdot\left(k_{m} \nabla T\right)=0
$$

At the mold-polymer boundary, the cycle averaged heat flux is used as boundary condition. The cycle averaged heat flux $\overline{q_{p}}$ is defined in equation (12), in which $t_{c}$ is the cycle time, $q(t)$ is the instantaneous heat flux [1].

$$
\overline{q_{p}}=\frac{1}{t_{c}} \int_{0}^{t_{c}} q(t) d t
$$

The instantaneous heat flux $q(t)$ can be obtained by solving the three-dimensional transient governing equation which is defined in equation (1) for plastic part only, and the boundary condition in equation (3) is applied to the part surface, but the mold temperature $T_{m}$ is assumed to be known from the previous iteration. Once the transient part temperature is obtained, $q(t)$ can be calculate by equation (13), in which $k_{p}$ is the thermal conductivity of the part, and $n$ is the normal of the 
part surface [1].

$$
q(t)=-k_{p} \frac{\partial T}{\partial n}
$$

At the mold-mold boundary, mold-coolant boundary and mold-air boundary, the treatment is the same with equation (6) to (10) in transient simulation.

\section{Mold-polymer and mold-mold boundaries treatment}

The normal FEM procedure, when discretizing equation (1), results in a sparse symmetric positive definite system matrix, such kind of matrices can be easily solved with the conjugate gradient solvers. However, in section 3.2 and 3.3 when dealing with the mold-mold boundary and mold-polymer boundary, the system matrix may violate the symmetric property if the meshes at the contact region are non-conformal. Figure 4 demonstrates the conformal mesh (A) and nonconformal mesh (B) in two-dimensional space for a more intuitive understanding, and it can be easily extended to three-dimensional tetrahedral mesh of this work. In conformal mesh, the nodes at the contact region are exactly one-one match, such as the red nodes and green nodes in the conform mesh plot. However, in non-conformal mesh there is no such property.

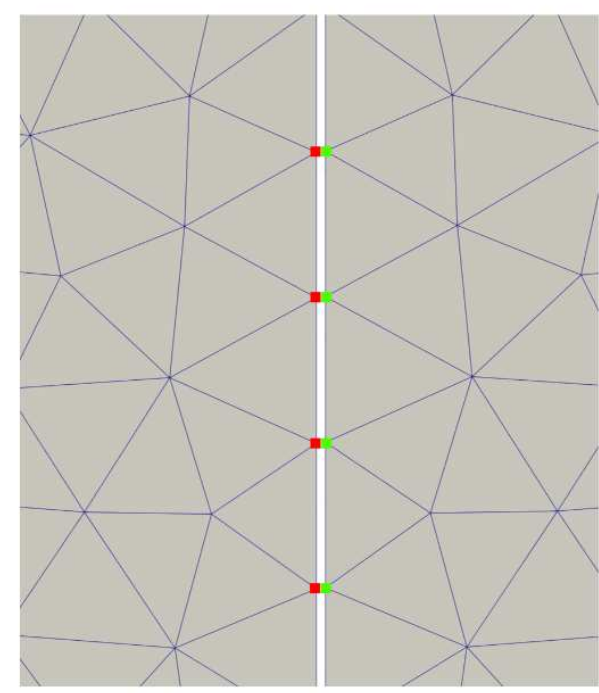

A - Conformal mesh

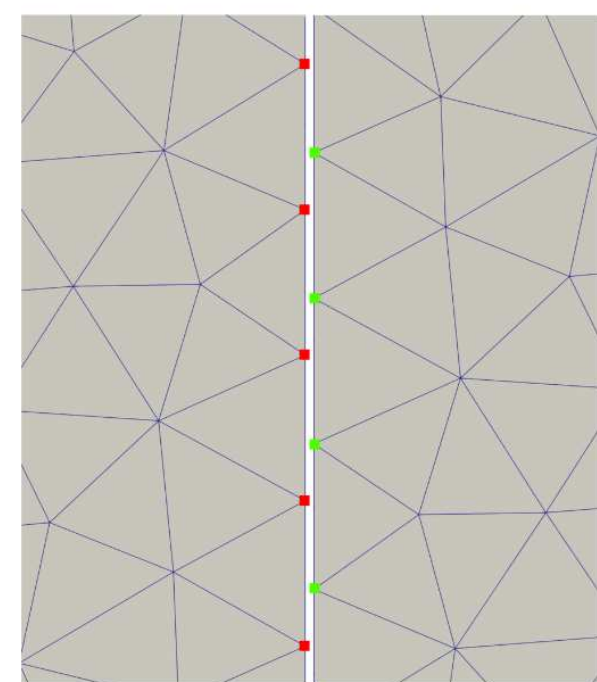

B - Non-conformal mesh

Figure 4. Conformal mesh and non-conformal mesh

Recall equation (3) for the three-dimensional tetrahedral conformal mesh shown in figure 5. $N_{p}$ is the FEM node on the plastic part surface, $S_{p}$ is the surface area of node $N_{p}, N_{m}$ is the FEM node on the mold surface, and $S_{m}$ is the surface area of node $N_{m}$. As the mesh is conformal at the mold-polymer boundary, we have $N_{p}$ is coincident with $N_{m}$, and $S_{p}$ equals to $S_{m}$. Integral equation (3) for node $N_{p}$ yields $h_{p} \cdot S_{p} \cdot\left(T_{N_{p}}-T_{N_{m}}\right)$, hence $K_{p m}=h_{p} \cdot S_{p}$, integral equation (3) for node $N_{m}$ yields $K_{m p}=h_{p} \cdot S_{m}$. The system matrix in equation (5) is still symmetric positive definite as $K_{p m}=K_{m p}$. 


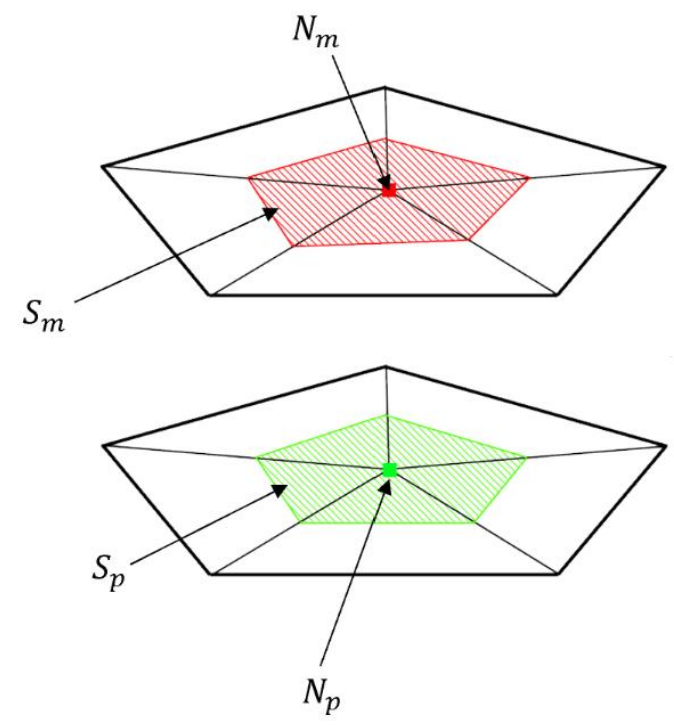

Figure 5. Conformal mesh treatment at the contact surface

Recall equation (3) for the non-conformal mesh shown in figure 6 , as the mesh is nonconformal at the contact region. The projection of polymer node $N_{p}$, which is denoted as $N_{p}{ }^{\prime}$, must be inside one surface triangle of the tetrahedral element. The three surface nodes of this surface mold tetrahedral element are $N_{m 1}, N_{m 2}$ and $N_{m 3} . S_{m 1}, S_{m 2}$ and $S_{m 3}$ are the areas of as indicated in figure 6. Integral equation (3) for plastic node $N_{p}$ yields $h_{p} \cdot S_{p} \cdot\left(T_{N_{p}}-\right.$ $\left.\frac{S_{m 1}}{\left(S_{m 1}+S_{m 2}+S_{m 3}\right)} T_{N_{m 1}}-\frac{s_{m 2}}{\left(S_{m 1}+S_{m 2}+S_{m 3}\right)} T_{N_{m 2}}-\frac{s_{m 3}}{\left(S_{m 1}+S_{m 2}+S_{m 3}\right)} T_{N_{m 3}}\right) . K_{p m}$ no longer equals to $K_{m p}$ under non-conformal mesh, hence the system matrix in equation (5) is asymmetric.
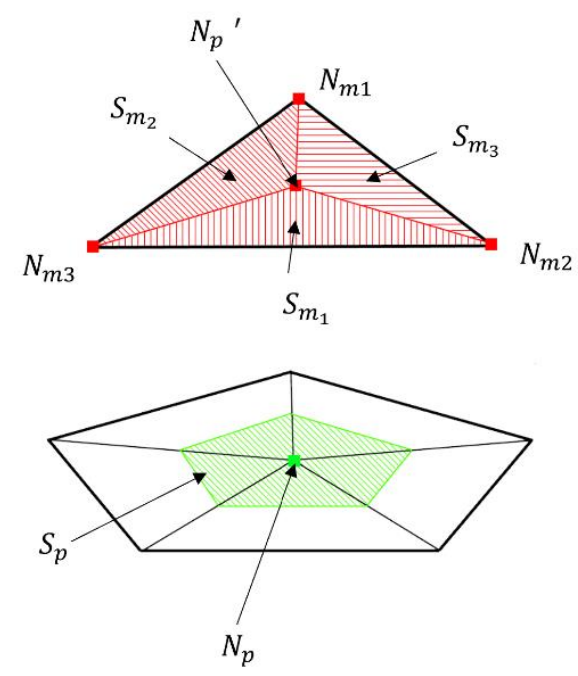

Figure 6. Non-conformal mesh treatment at the contact surface

Although conformal mesh brings better properties, it is too strict and not practical in complex situations. So, in this work we adopted the non-conformal mesh. Non-conformal mesh generates asymmetric matrix which can't be solved with the normal conjugate gradient solver, instead the 
BiCGSTAB method $[20]$ is used to solve such asymmetric matrix.

\section{Experiment and Simulation Setup}

An injection molding mold was designed for validation purpose as shown in figure 7 . The injection molding experiments were performed on a JSW J110ADC-180H electric injection molding machine, and two Kistler 6190CA sensors were embedded at the mold surface of the runner system. This is a two cavities configuration, in which a pair of plastic parts can be made in one single shoot.
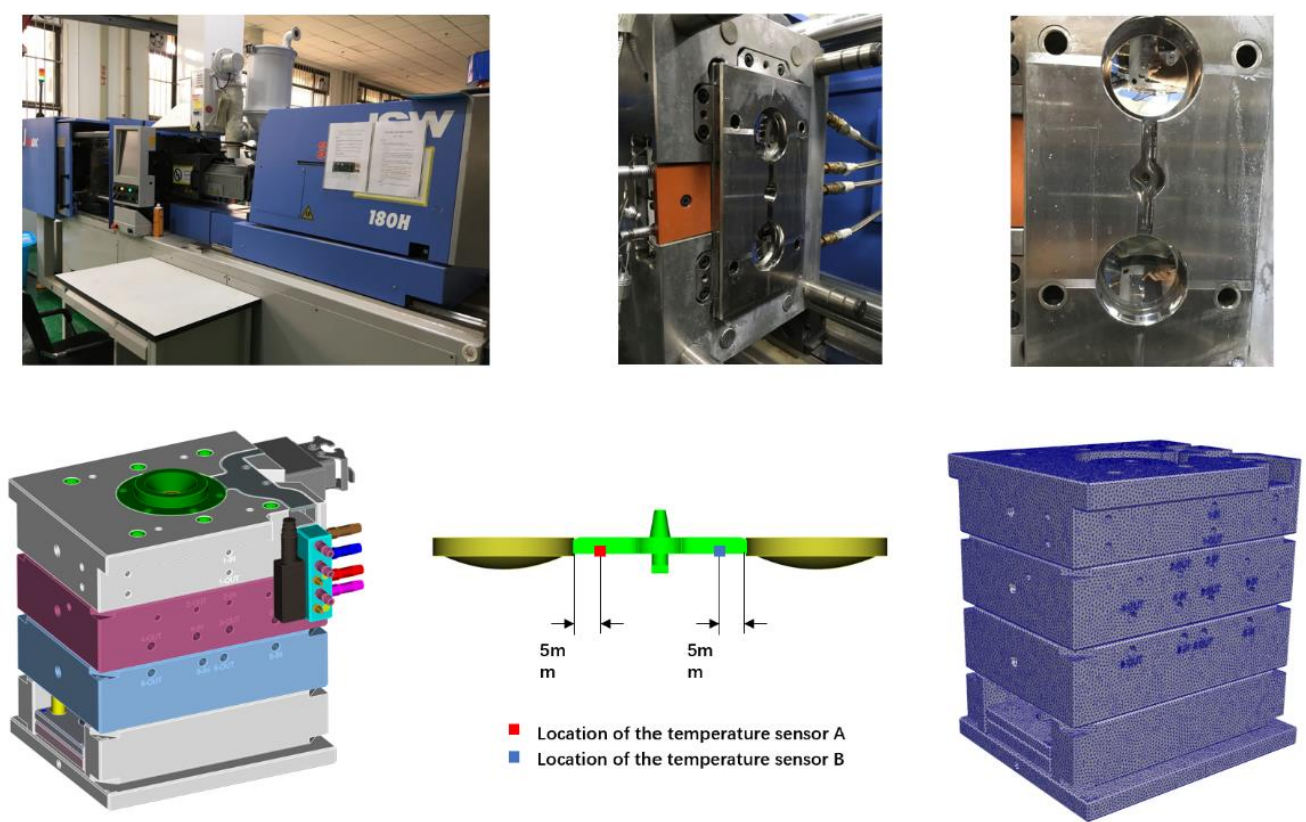

Figure 7. The model of the validation mold

The plastic material for injection molding is SABIC polycarbonate OQ2720. Table 1 and table 2 list the material thermal properties and PVT properties used in transient cooling analysis.

Table 1. Material thermal properties.

\begin{tabular}{lcc}
\hline Material thermal properties & Temperatures & Values \\
\hline Specific heat $\left(\mathrm{J} \cdot \mathrm{K}^{-1} \cdot \mathrm{Kg}^{-1}\right)$ & $330{ }^{\circ} \mathrm{C}$ & 1880 \\
Thermal conductivity $\left(\mathrm{W} \cdot \mathrm{K}^{-1} \cdot \mathrm{m}^{-1}\right)$ & $50{ }^{\circ} \mathrm{C}$ & 0.25 \\
& $100{ }^{\circ} \mathrm{C}$ & 0.25 \\
& $150{ }^{\circ} \mathrm{C}$ & 0.27 \\
& $200{ }^{\circ} \mathrm{C}$ & 0.22 \\
& $250{ }^{\circ} \mathrm{C}$ & 0.23 \\
& $300{ }^{\circ} \mathrm{C}$ & 0.24 \\
\hline
\end{tabular}

Table 2. Material PVT model.

\begin{tabular}{lc}
\hline Material PVT model & Values \\
\hline $\mathrm{b} 5(\mathrm{~K})$ & 418.93 \\
$\mathrm{~b} 6\left(\mathrm{~K}^{\cdot} \mathrm{Pa}^{-1}\right)$ & $3.21 \mathrm{e}-07$ \\
\hline
\end{tabular}




\begin{tabular}{lc}
\hline $\mathrm{b} 1 \mathrm{~m}\left(\mathrm{~m}^{3} \cdot \mathrm{Kg}^{-1}\right)$ & 0.0008654 \\
$\mathrm{~b} 2 \mathrm{~m}\left(\mathrm{~m}^{3} \cdot \mathrm{Kg}^{-1} \cdot \mathrm{K}^{-1}\right)$ & 0.0008654 \\
$\mathrm{~b} 3 \mathrm{~m}(\mathrm{~Pa})$ & $1.73897 \mathrm{e}+08$ \\
$\mathrm{~b} 4 \mathrm{~m}\left(\mathrm{~K}^{-1}\right)$ & 0.003462 \\
$\mathrm{~b} 1 \mathrm{~s}\left(\mathrm{~m}^{3} \cdot \mathrm{Kg}^{-1}\right)$ & 0.0008612 \\
$\mathrm{~b} 2 \mathrm{~s}\left(\mathrm{~m}^{3} \cdot \mathrm{Kg}^{-1} \cdot \mathrm{K}^{-1}\right)$ & $5.85 \mathrm{e}-08$ \\
$\mathrm{~b} 3 \mathrm{~s}(\mathrm{~Pa})$ & $3.4343 \mathrm{e}+08$ \\
$\mathrm{~b} 4 \mathrm{~s}\left(\mathrm{~K}^{-1}\right)$ & 0.00227 \\
$\mathrm{~b} 7\left(\mathrm{~m}^{3} \cdot \mathrm{Kg}^{-1}\right)$ & 0 \\
$\mathrm{~b} 8\left(\mathrm{~K}^{-1}\right)$ & 0 \\
$\mathrm{~b} 9\left(\mathrm{~Pa}^{-1}\right)$ & 0 \\
\hline
\end{tabular}

For the mold side, the mold material properties and the actual injection molding conditions are given in table 3 and table 4 . The CAD assembly of this injection mold has 142 components, after removing some small unimportant features, there are $12 \mathrm{CAD}$ mold components used to generate three-dimensional tetrahedral mesh for transient cooling analysis as shown in figure 7. The mesh statistics shows that there are 6,924,353 tetrahedral mesh. Among the 6,924,353 mesh, there are 742,047 tetrahedral mesh for the plastic part, and the rest 6,182,306 elements are the mold meshes.

Table 3. Mold material properties.

\begin{tabular}{lc}
\hline Material thermal properties & Values \\
\hline Specific heat $\left(\mathrm{J} \cdot \mathrm{K}^{-1} \cdot \mathrm{Kg}^{-1}\right)$ & 460 \\
Thermal conductivity $\left(W \cdot \mathrm{K}^{-1} \cdot \mathrm{m}^{-1}\right)$ & 29 \\
Density $\left(\mathrm{Kg} \cdot \mathrm{m}^{-3}\right)$ & 7800 \\
\hline
\end{tabular}

Table 4. Molding conditions.

\begin{tabular}{lc}
\hline Molding conditions & Values \\
\hline Melt temperature $\left({ }^{\circ} \mathrm{C}\right)$ & $290 / 300 / 320$ \\
Coolant temperature $\left({ }^{\circ} \mathrm{C}\right)$ & 110 \\
Injection + packing + cooling time $(\mathrm{s})$ & 70 \\
Mold open time $(\mathrm{s})$ & 5 \\
Coolant flowrate $\left(\mathrm{m}^{3} \cdot \mathrm{s}^{-1}\right)$ & $4 \times 10^{-4}$ \\
Heat transfer coefficient, mold-polymer $\left(\mathrm{W} \cdot \mathrm{K}^{-1} \cdot \mathrm{m}^{-2}\right)$ & 2500 \\
Heat transfer coefficient, mold-mold $\left(\mathrm{W} \cdot \mathrm{K}^{-1} \cdot \mathrm{m}^{-2}\right)$ & 30000 \\
\hline
\end{tabular}

\section{Results and Discussion}

The experiment performed injection molding trials under three different initial melt temperatures: 320 degrees, 305 degrees, and 290 degrees. For each initial melt temperature condition, three experiments were performed and the actual temperature data was collected. Figure 8 shows the simulation temperature results and the three experimental temperature results on sensor A and B for 320 degrees melt temperature, "sim" stands for simulation, "exp-1", "exp-2" and "exp- 
3" stand for the three experiments. The left side of figure 8 plots the simulated temperature and the actual temperatures from the temperature sensors, we can see the simulation matches experiments well from both trend and magnitude perspective. The in-cycle mold temperature starts at a relative low value, when the injection + packing starts, the mold temperature increases quickly and then cools down gradually to the low temperature similar with the beginning of the cycle. The right side of figure 8 plots the temperature error between simulation and experiments at each time step, the experimental mold temperature used for this plot is the averaged mold temperature over the three runs, then calculate the error in percentage against the simulation result for each time step, we can see that the maximum error is $3.79 \%$. Figure 9 and 10 plot the same results for the initial melt temperature under 305 degrees and 290 degrees. The maximum error and average error for this experiment are listed in table 5 .
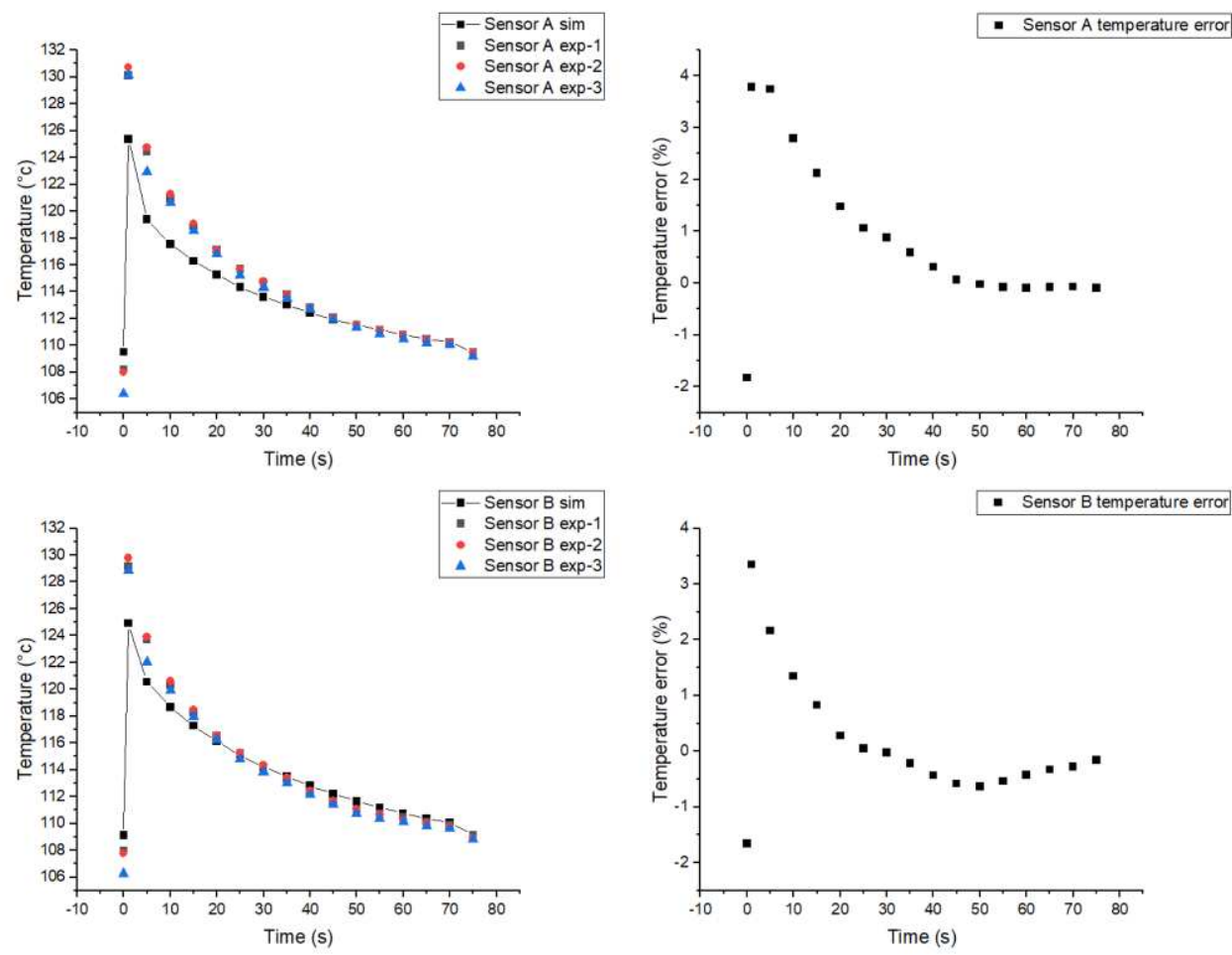

Figure 8. Validation data for melt temperature 320 degrees 

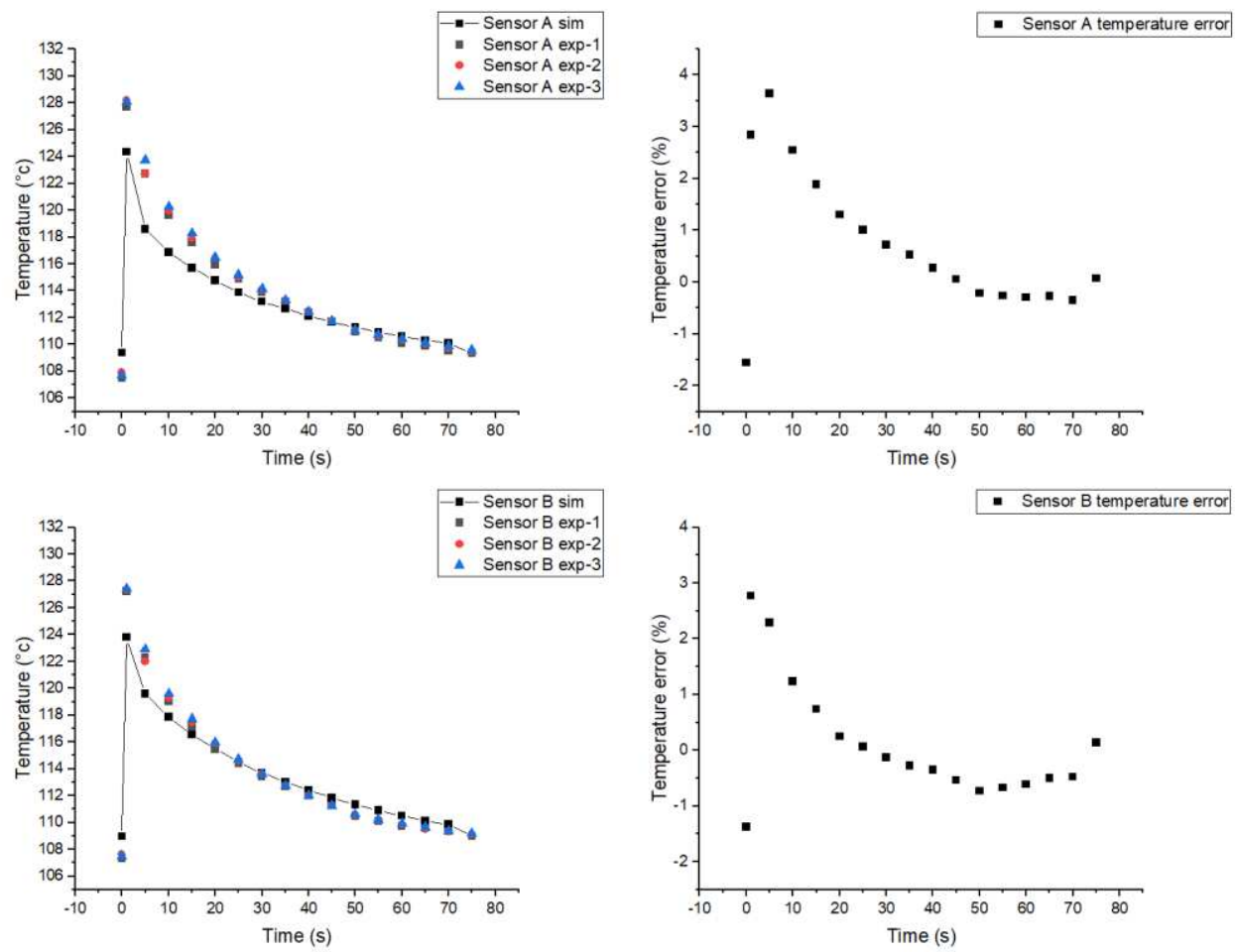

Figure 9. Validation data for melt temperature 305 degrees
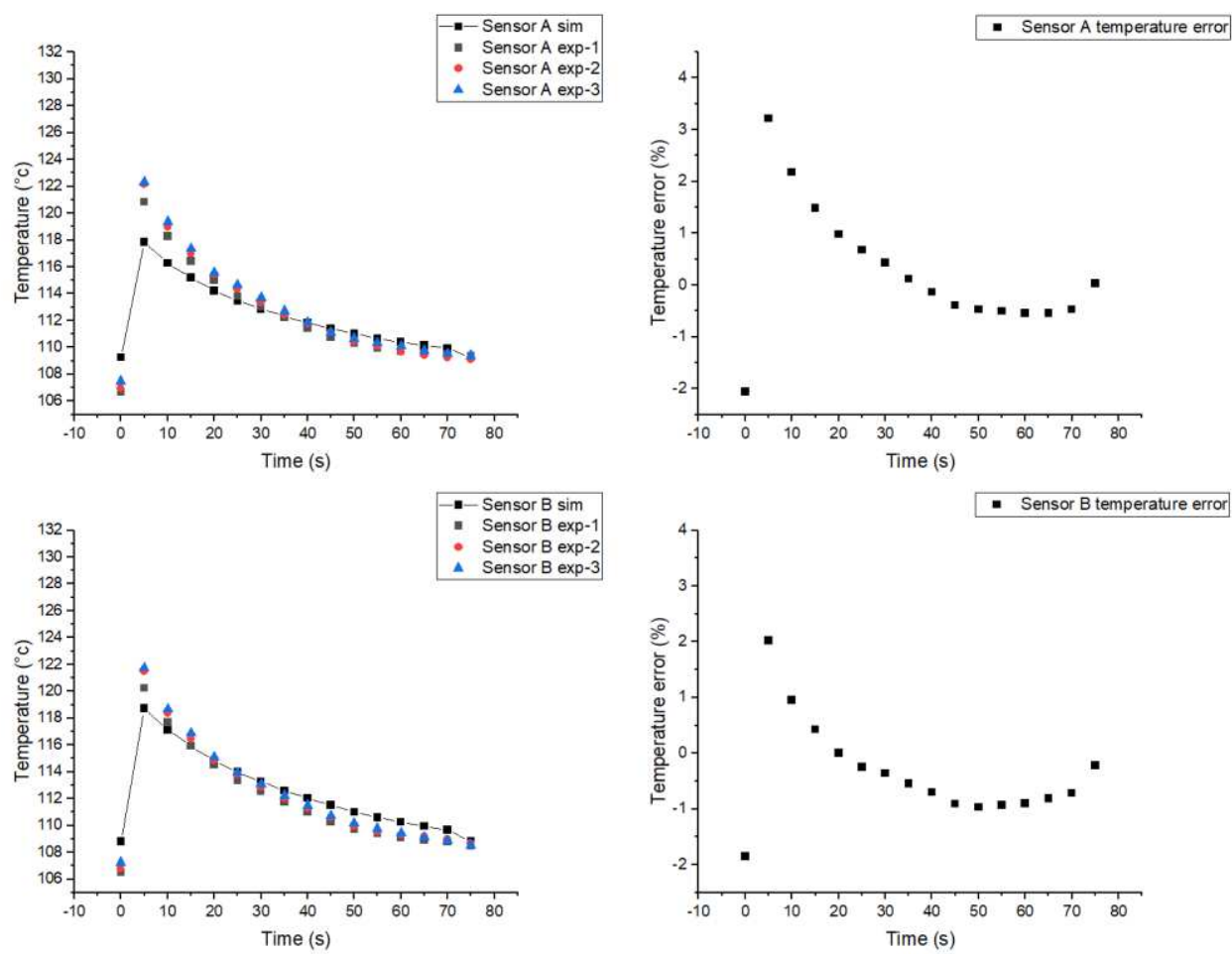

Figure 10. Validation data for melt temperature 290 degrees 
Table 5. Simulation accuracy for initial melt temperatures 320, 305 and 290 degrees.

\begin{tabular}{ccc}
\hline Initial melt temperature $\left({ }^{\circ} \mathrm{C}\right)$ & Maximum error $(\%)$ & Average error $(\%)$ \\
\hline 320 & 3.79 & 0.95 \\
305 & 3.64 & 0.91 \\
290 & 3.22 & 0.84 \\
\hline
\end{tabular}

The polymer and mold are not in perfect contact according to [19]. The mold temperature and part temperature are not equal at the mold-polymer boundary, as there is a heat resistance at the mold-polymer boundary. This work uses the heat transfer coefficient $h_{p}$ to model such thermal behavior. Larger $h_{p}$ means smaller heat resistance, then the polymer temperature and mold temperature at the mold-polymer boundary are closer to each other, and vice versa. In this section we will investigate the effect of the heat transfer coefficient at mold-polymer boundary with $\mathrm{h}_{\mathrm{p}}=$ $2500 \mathrm{~W} \cdot \mathrm{K}^{-1} \cdot \mathrm{m}^{-2}$, and check temperature difference between the mold temperature and its contacted plastic part temperature. The locations to check are selected at the center of cavity (locations B and D) as shown in figure 11.

Similar with the treatment at the mold-polymer boundary, the heat transfer coefficient $h_{m}$ is also applied at the mold-mold boundary. However, unlike the mold-polymer boundary, the heat resistance between the mold-mold boundary is smaller. In this work the heat transfer coefficient at the mold-mold boundary is set as $\mathrm{h}_{\mathrm{m}}=30000 \mathrm{~W} \cdot \mathrm{K}^{-1} \cdot \mathrm{m}^{-2}$. Figure 11 indicates the location of the two checkpoints which are at the contact region between mold core platen and mold cavity platen (locations A and C). We use those two locations to exam the heat resistance implementation at moldmold boundary by checking their temperature difference.

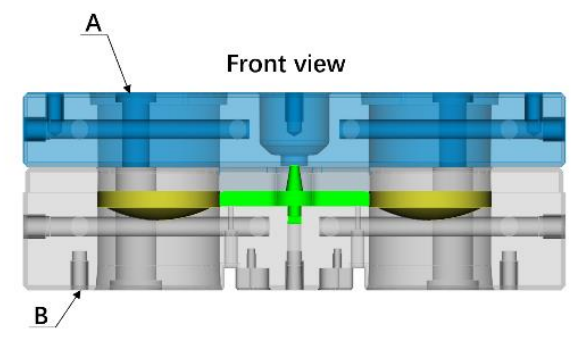

A - Mold core platen B - Mold cavity platen

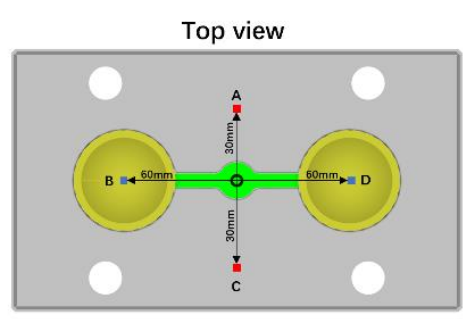

A/C - Mold-mold boundary B/D - Mold-cavity boundary

Figure 11. Mold-mold/mold-polymer temperature difference check points

The figure 12 plots temperature difference at the boundaries within the cycle for location A, B, $\mathrm{C}$ and D. Clearly, we can see that the temperature differences for $\mathrm{B}$ and $\mathrm{D}$ are much larger than locations $\mathrm{A}$ and $\mathrm{C}$. This is because the locations $\mathrm{B}$ and $\mathrm{D}$ are at the mold-polymer boundary, where we set smaller heat transfer coefficient. Hence the heat conduction from hot polymer into the mold through the contact boundary is slower due to larger interface heat resistance. The temperature differences for $\mathrm{B}$ and $\mathrm{D}$ are larger than 200 degrees at the begin of the cycle, that is when the initial melt temperature is 320 degrees while the mold is around 110 degrees. Because of this heat resistance effect, the mold temperature doesn't jump to the polymer temperature instantaneously, 
but rather the temperature difference decreases gradually, and finally the difference is approaching zero at the end of the cycle. Such temperature gap transition means the heat resistance effect has been correctly modeled in the simulation. The temperature difference for locations $\mathrm{A}$ and $\mathrm{C}$ are less than 0.3 degrees, which is much smaller than locations $\mathrm{B}$ and $\mathrm{D}$. This is because locations $\mathrm{A}$ and $\mathrm{C}$ are at the mold core and mold cavity contact interface, where the heat transfer coefficient is larger and smaller heat resistance at the thermal contact interface.
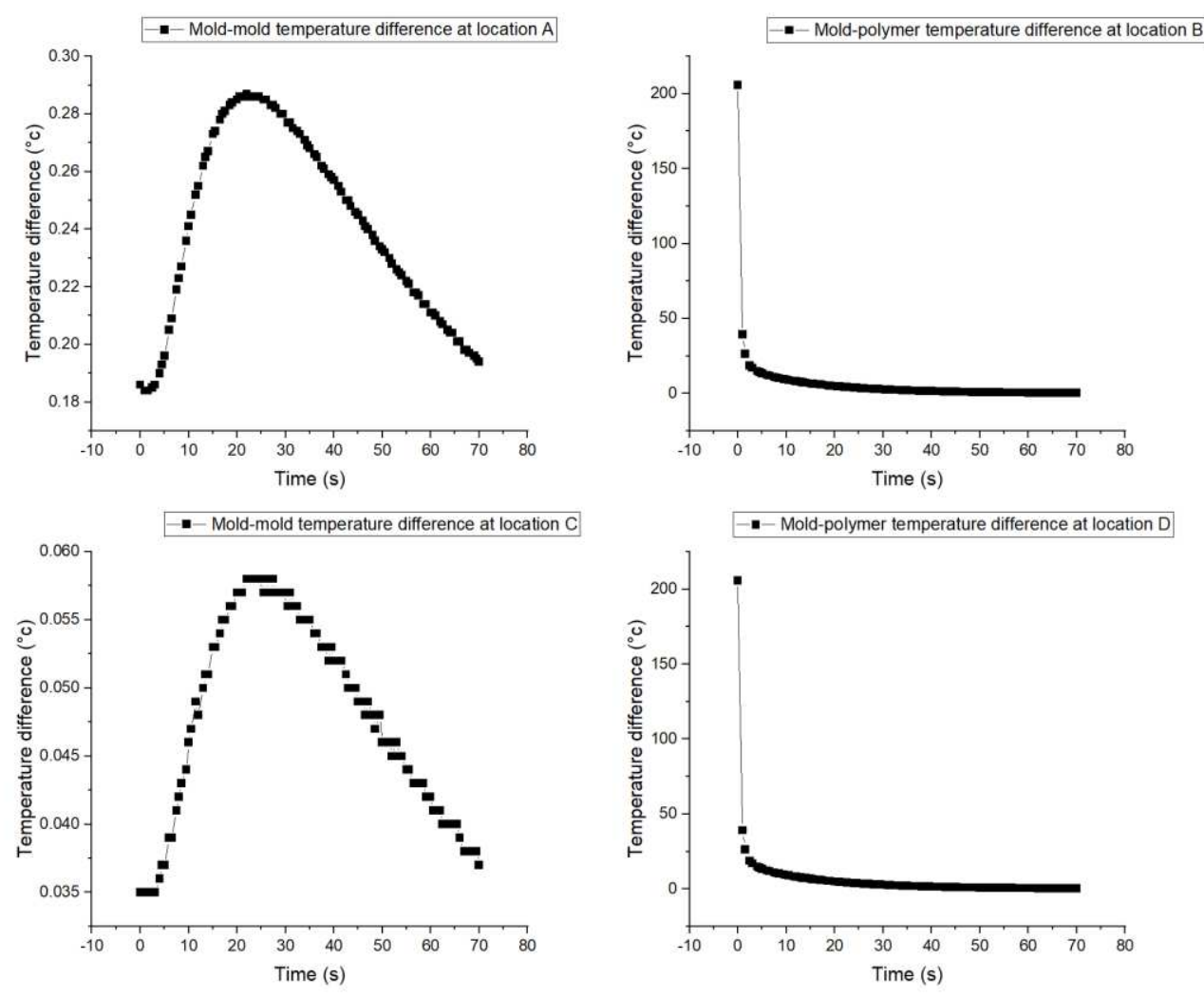

Figure 12. Mold-mold/mold-polymer temperature difference plot

Figure 13 shows the transient mold temperatures under the stable cycle, under the molding condition that the initial melt polymer temperature is 320 degrees. All the plots in figure 13 are rendered in the open-sourced software ParaView. From the plots we can see that the highest temperature appears at the mold core side which is directly in contact with the melt polymer, the mold temperature first increases at the beginning of the injection cycle, then the temperature drops down. It should be noted that the temperature of top half mold is higher than the bottom half mold. The temperature of top half mold is slightly lower than 110 degrees, but the bottom half mold is much lower than 110 degrees. There are 4 cooling channels at the top half mold, but only 1 cooling channel at the bottom half, and the cooling channel inlet temperature is 110 degrees. This means that the coolant actually heats up the mold and maintains the mold temperature at about 110 degrees. 

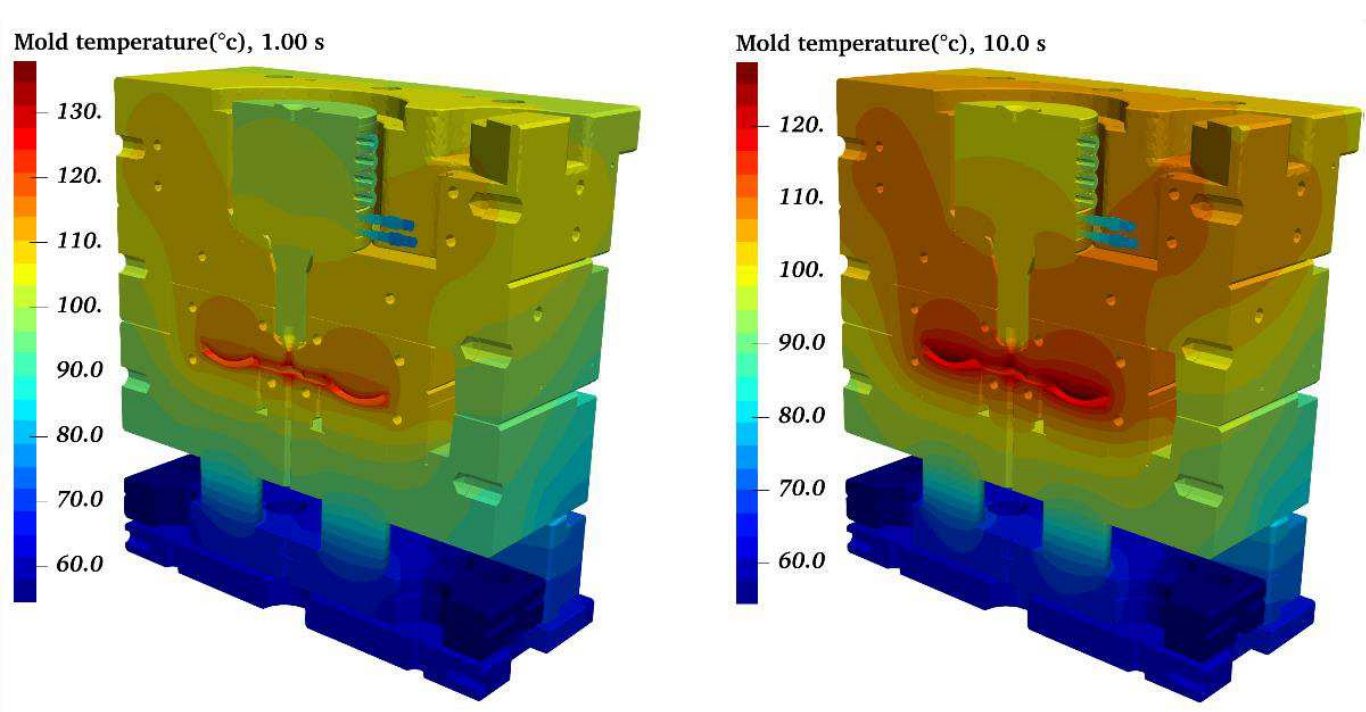

Mold temperature $\left({ }^{\circ} \mathrm{c}\right), 25.0 \mathrm{~s}$
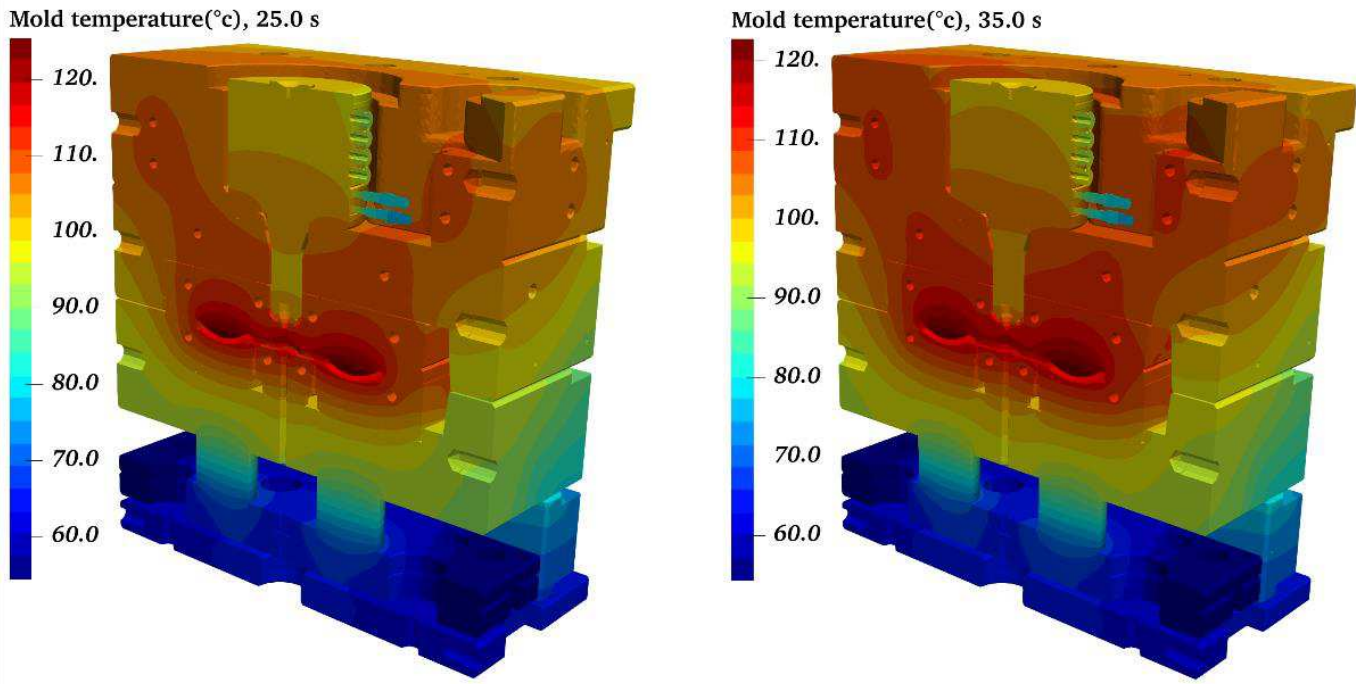

Mold temperature $\left({ }^{\circ} \mathrm{c}\right), 75.0 \mathrm{~s}$
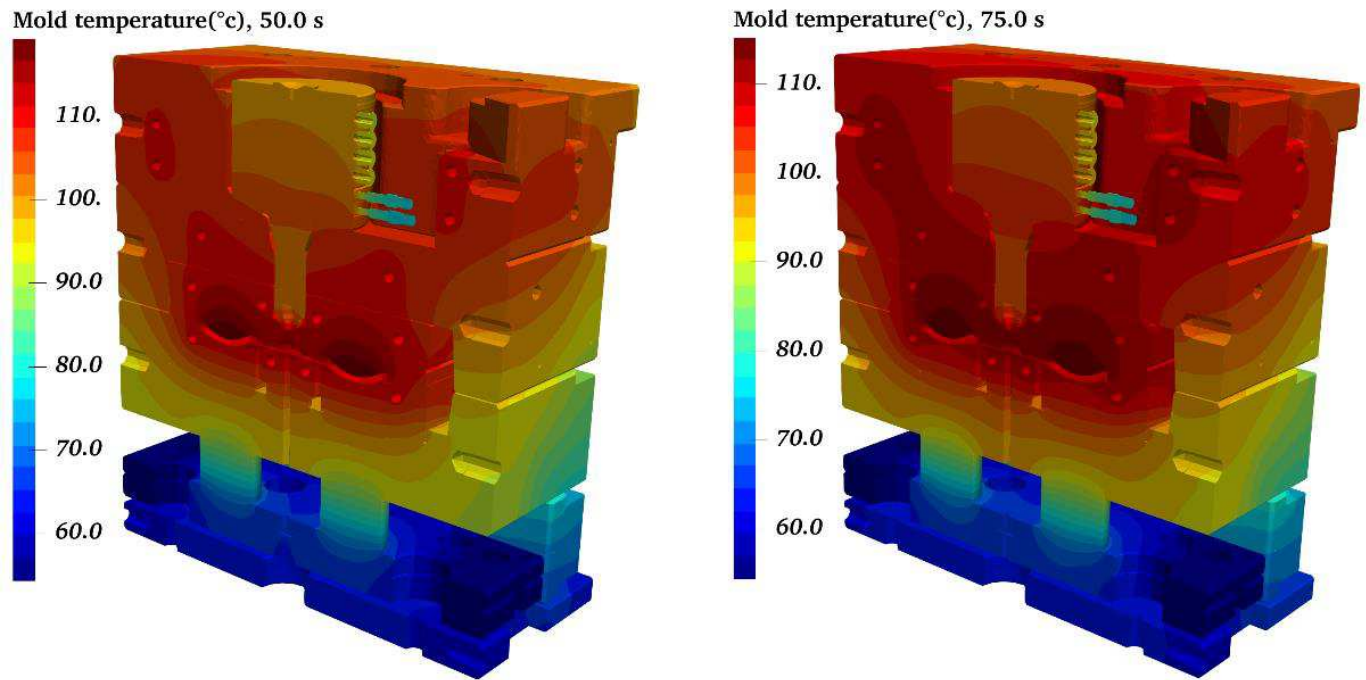

Figure 13. The transient mold temperatures at the stable cycle.

Figure 14 shows the transient plastic part temperature under the stable cycle at different time steps. From the plots we can clearly see the part cools down from surface region to the core region at the end of the injection cycle. At the end of the injection molding cycle, the plastic part 
temperature drops down to around 110 degrees, which the coolant inlet temperature.
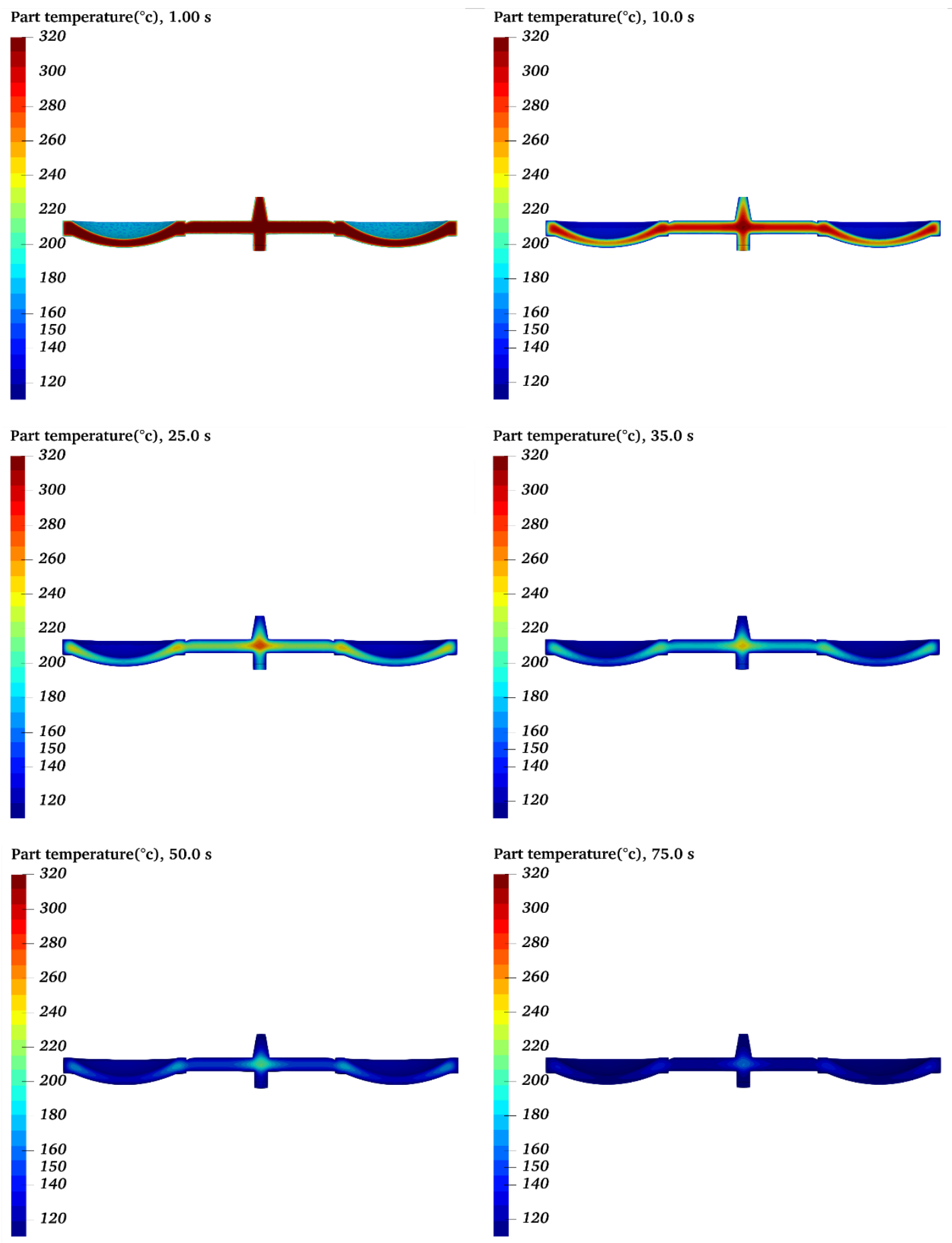

Figure 14. The transient part temperatures at the stable cycle.

This work also performed two extra analyses: (1) transient cooling simulation with a uniform mold temperatures (40 degrees) as initial condition; (2) cycle averaged cooling simulation, which is actually the initial condition in chapter 4 . These two extra simulations are used to compare against the transient cooling simulation presented in this work. The metrics used are the execution time and the average cavity temperature. For the two transient simulations, the average cavity temperature is the average temperature over all the mold nodes at the mold-polymer boundary through the stable 
injection molding cycle; For the cycle averaged cooling simulation, the average cavity temperature is the average temperature over all the mold nodes at the mold-polymer boundary. Such definition on the average cavity temperature makes the comparison between transient simulation and steady state simulation possible.

From table 6 we can see that all these three simulations are at the same level of accuracy: the average cavity temperature error between the two transient FEM methods is $0.15 \%$, and the error between the cycle averaged cooling simulation and the transient cooling simulation is $0.2 \%$. Such observation proofs the definitions of figure 1 , hence we can conclude that the initial condition proposed in chapter 4 has no negative impact to the simulation accuracy. When comparing the execution time, we can see that the FEM with solved initial condition is more than 14 times faster than the FEM with uniform initial condition, which is a significant speed acceleration. Although it is 4.3 times slower than the cycle averaged cooling simulation, it is still satisfactory considering it takes less than 8 minutes to finish.

Table 6. The execution time and average mold cavity temperature comparison

\begin{tabular}{lcc}
\hline \multicolumn{1}{c}{ Method } & $\begin{array}{c}\text { Execution time } \\
(\mathbf{s})\end{array}$ & $\begin{array}{c}\text { Average cavity temperature } \\
\left.\left({ }^{\circ} \mathrm{C}\right)\right)\end{array}$ \\
\hline FEM with solved initial condition & 478 & 113.05 \\
FEM with uniform initial condition & 6707 & 112.88 \\
Cycle averaged cooling simulation & 110 & 113.28 \\
\hline
\end{tabular}

\section{Conclusions}

Conventional cooling simulation for injection molding uses the BEM to perform the cycleaveraged steady state analysis. Using cycle-averaged temperature to approximate the transient mold temperature loses accuracy, especially such approximation won't work for some special injection molding processes. The advantage of BEM is that it reduces the spatial dimensions of the computational domain to the mold surfaces, however, the cost is BEM generates a dense, asymmetric system matrix, which is memory consuming and takes very long time to solve. This paper introduces the FEM based transient cooling simulation method for injection molding, which leverages the advantage of FEM and uses cycle-averaged solution as initial condition. This method obtains the transient mold temperature with a quite satisfactory computation time and adequate accuracy. Although this work only discusses for the injection molding process, it can be easily extended to other molding processes such as the compression molding process and gas assisted molding process.

\section{Declarations}

Funding: This research was funded by the National Natural Science Foundation of China, Grant Nos. U20A20288.

Conflicts of interest/Competing interests: The authors have no relevant financial or nonfinancial interests to disclose. 
Availability of data and material: All data and models generated or used during the study appear in the submitted manuscript.

Code availability: All code generated or used during the study appear in the submitted manuscript.

Authors' contributions: Zhigao Huang conceived the idea; Zhigao Huang and Lu Chen conducted the analyses; Lu Chen and Xiaowei Zhou participated in the development and testing of the prototype system; all authors contributed to the writing of the manuscript. 


\section{References}

1. Zhou H, Zhang Y, Wen J, Li D (2009) An acceleration method for the BEM-based cooling simulation of injection molding. Engineering analysis with boundary elements 33(8-9):1022-30.

2. Kwon TH (1988) Mold Cooling System Design Using Boundary Element Method. Journal of Engineering for Industry 110(4):384-94.

3. Chen S-C, Hu S-Y (1991) Simulations of cycle-averaged mold surface temperature in mold-cooling process by boundary element method. International communications in heat and mass transfer 18(6):823-32.

4. Koo BH, Choi KI (2005) Three-Dimensional Mold Cooling Analysis for Injection Molding Process. Society of Plastics Engineers Annual Technical Conference 2:103.

5. Chen S, Huang S, Jiang Z, Li H, Shen C (2005) 3D simulation and verification for mold temperature control technologies. Society of Plastics Engineers Annual Technical Conference 2:74.

6. Qiao H (2006) A systematic computer-aided approach to cooling system optimal design in plastic injection molding. International journal of mechanical sciences 48(4):430-439.

7. Zhou H, Li D (2005) Mold cooling simulation of the pressing process in TV panel production. Simulation Modelling Practice and Theory 13(3):273-285.

8. Chiang H, Himasekhar K, Santhanam N, Wang K (1993) Integrated simulation of fluid flow and heat transfer in injection molding for the prediction of shrinkage and warpage. Journal of Engineering Materials and Technology 115(1):37-47.

9. Rezayat M, Burton TE (1990) A boundary-integral formulation for complex three-dimensional geometries. International Journal for numerical methods in engineering 29(2):263-273.

10. Park SJ, Kwon TH (1998) Thermal and design sensitivity analyses for cooling system of injection mold, Part 1: thermal analysis. Journal of Manufacturing Science and Engineering 120(2):287-295.

11. Park SJ, Kwon TH (1998) Thermal and Design Sensitivity Analyses for Cooling System of Injection Mold, Part 2: Design Sensitivity Analysis. Journal of Manufacturing Science and Engineering 120(2):296-305.

12. Park SJ, Kwon TH (1998) Optimization method for steady conduction in special geometry using a boundary element method. International journal for numerical methods in engineering 43(6):11091126.

13. Hioe Y, Chang KC, Zuyev K, Bhagavatula N, Castro JM (2008) A simplified approach to predict part temperature and minimum "safe" cycle time. Polymer Engineering \& Science 48(9):1737-1746.

14. Hu S, Cheng N, Chen S (1995) Effect of cooling system design and process parameters on cyclic variation of mold temperatures--simulation by DRBEM. Plastics, Rubber \& Composites Processing and Appl 4(23):221-232.

15. Tang LQ, Pochiraju K, Chassapis C, Manoochehri S (1996) Three-dimensional transient mold cooling analysis based on galerkin finite element formulation with a matrix-free conjugate gradient technique. International Journal for Numerical Methods in Engineering 39(18):3049-3064.

16. Gaul L, Kögl M, Wagner M (2003) Introduction. In: Boundary element methods for engineers and scientists: an introductory course with advanced topics, 1st ed. Springer-Verlag Berlin Heidelberg, Berlin, Germany, pp. 11-16.

17. Fetecau C, Dobrea D, Postolache I (2010) Overmolding injection molding simulation of tensile test specimen. International Journal of Modern Manufacturing Technologies 2(2):45-50.

18. Sánchez, R, Martinez A, Mercado D, Carbonel A, Aisa J (2021) Rapid heating injection moulding: An experimental surface temperature study. Polymer Testing 93:106928.

19. Liu Y, Gehde M (2015) Evaluation of heat transfer coefficient between polymer and cavity wall for improving cooling and crystallinity results in injection molding simulation. Applied Thermal Engineering 80:238-246.

20. Van d VHA B (1992) BI-CGSTAB: a fast and smoothly converging variant of BI-CG for the solution of nonsymmetric linear systems. Siam Journal on Scientific \& Statistical Computing 13(2):631-644. 